\title{
Gestión del Talento Humano: Análisis desde el Enfoque Estratégico
}

\author{
Reynier I. Ramírez(1), Cesar A. Espindola(2), Gladis I. Ruíz ${ }^{(3)}$ y Alfredo M, Hugueth(1) \\ (1) Universidad de la Costa, Dpto. de Ciencias Empresariales, Atlántico - Colombia. \\ (e-mail: rramirez13@cuc.edu.co; ahugueth1@cuc.edu.co) \\ (2) Universidad de La Serena, Fac. de Ingeniería, Dpto. de Ing. Industrial, La Serena - Chile. \\ (e-mail: cespindola@userena.cl) \\ (3) Universidad Simón Bolívar, Atlántico - Colombia. (e-mail: gladis.ruiz@unisimonbolivar.edu.co)
}

Recibido Feb. 21, 2019; Aceptado Abr. 11, 2019; Versión final May. 15, 2019, Publicado Dic. 2019

\section{Resumen}

El objetivo del trabajo fue el análisis de la gestión del talento humano desde el enfoque estratégico en el sector minero de Venezuela, Colombia y Chile. Se emplea un método cuantitativo, tipo descriptivo, y diseño no experimental transversal. Se emplea un cuestionario de nueve ítems, aplicado a 55 gerentes, adscritos a cada vice presidencia de las empresas Carbones del Guasare, S.A, Complejo Carbonífero de Cerrejón y Mina Invierno. Los resultados evidenciaron diferencias altamente significativas considerándose moderadamente óptimo, predominando la estrategia organizacional, seguido de los rasgos y tendencia actual, con falencias en el modelo funcional. El estudio muestra que en el sector minero de Venezuela, Colombia y Chile se le da prioridad a la estrategia organizacional, determinado por el indicador socialización de las actividades laborales.

Palabras clave: gestión del talento; enfoque estratégico; gestión estratégica, talento humano

\section{Human Talent Management: Analysis from the Strategic Approach}

\begin{abstract}
The objective of the study was the analysis of human talent management from the strategic approach in the mining sector of Venezuela, Colombia and Chile. A quantitative, descriptive, transversal, non-experimental design approach is employed. A nine-item questionnaire was applied to 55 managers, attached to each vice presidency of the companies Carbones del Guasare, S.A, Cerrejón and Winter carbon mine. The results showed highly significant differences being moderately optimum, highlighting the organizational strategy, followed by the features and current trend, with flaws in the functional model. The study shows that the mining sector in Venezuela, Colombia and Chile gives especial attention to the organizational strategy, determined by the indicator socialization of labor activities.
\end{abstract}

Keywords: talent management; strategic approach; strategic management; human talent 


\section{INTRODUCCIÓN}

La gestión de talento humano(GTH), en los primeros años, indicaban el estado alcanzado de actividades ejecutadas por el personal, permaneciendo inalterables a pesar que la gestión organizacional ha modificado su visión estratégica en estos últimos tiempos. Los enfoques de la gestión humana en el sector industrial están modificándose mundialmente, procede del entorno empresarial, transferencia de modelos y técnicas corporativas, lucrativo y sin ánimo de lucro, es una realidad del día tras días, experimentadas por las empresas carboníferas. Para Maxwell (2016), Cuesta (2015), Schuler y Tarique (2012) y Coff y Kryscynski (2011), parte del concepto que los seres humanos son talentos y no recursos de los que se pueden disponer a discreción, con posibilidades de desarrollo, aplicabilidad y aporte. Para quienes investigan, la GTH son acciones que ejecutan las personas, mediante sus competencias, en un área en específico, fortaleciendo procesos de desarrollo, proyección y mejores condiciones de sí mismo y colectivamente en un entorno organizacional. En América Latina, se percibió como una manera de trabajar con la gente, cuyo objetivo se basaba en el diagnóstico de funciones, evolucionando hasta convertirse en una herramienta sólida de dirección estratégica con el fin de conceder al líder, información necesaria que permita tener una orientación acertada para el accionar productivo de la organización.

De acuerdo a recientes estudios internacionales, realizados en el área de talento humano por ManpowerGroup y Deloitte (2018), se hace énfasis en el significado de la gestión y su impacto social tanto en el sector real como en sus medios externos, y la escasez de talento a nivel mundial, alcanzando el valor más alto de estos últimos 12 años, representando el $45 \%$. Ante las necesidades de nuevas habilidades, fuerza laboral que envejece, endurecimiento del mercado laboral, situaciones políticas, económicas y sociales que atraviesan las organizaciones, por lo que es necesario desarrollar estrategias que le permitan a la empresa desde el talento humano, poder consolidar sus metas.

En América del Sur, países como Argentina, Brasil, Ecuador y Perú, han implementado la GTH en sus organizaciones con estrategias de acompañamiento al personal, compensación y políticas de retención; generando un efecto positivo hacia la consecución de objetivos (Ramírez et al., 2018c). Esto patentiza que la puesta en marcha de elementos estratégicos organizacionales considerando un enfoque estratégico para la GTH con los rasgos y tendencias actuales son determinantes para la efectividad empresarial. En Venezuela, Colombia y Chile, la GTH está adaptándose a las condiciones específicas de cada empresa, aplicable al momento histórico, sistema socio-cultural del país. En el Estado Zulia - Venezuela, la Costa Atlántica de Colombia y Santiago de Chile, parte de las organizaciones se encuentran a las expectativas de gestionar el talento humano con un enfoque estratégico, utilizada para definir con precisión los objetivos que conducirán al desarrollo de la organización adaptándose a las necesidades del entorno, viéndose inmersos en este proceso de gestión gerencial, por tal motivo, se asesoran con especialistas para iniciar sus procesos de implementación para la gestión del talento humano desde el enfoque estratégico.

De allí que la gestión del talento humano desde el enfoque estratégico, responde al cómo aplicar los procesos del talento humano, decidiendo la estrategia organizacional (Ramírez et al., 2018e; Bryant y Allen, 2013), adaptándose a las mejores prácticas que se requieran para acompañar a la gente (Ramírez y Hugueth, 2017; Anaya, 2016), mediante la creación armónica de estructuras, principios y controles (Safa et al., 2017), buscando el desarrollo de la gente, centrándose en el futuro, sucesión auto gestionada, banco de datos de personas y gestión compartida (Caplan,2014), adecuándose a los desafíos derivados de los mercados emergentes (Villalobos y Ramírez 2018; Ambrosius,2016).

Al respecto, se puede decir que la gestión del talento humano consiste en desarrollar cualidades competitivas para implementar políticas y crear una estructura organizacional que sea favorable, conducente a una asignación de recursos que permita alcanzar con éxito esas estrategias. Permite anticipar y gerenciar colectivamente el cambio, para crear permanentemente estrategias que garanticen la razón de ser de la organización y su futuro. Las empresas del sector minero al desconocer las estrategias asociadas a la GTH, probablemente desmejoren la consecución favorable de sus resultados, que va más allá de la evaluación de los procesos administrativos comunes, imposibilitando las mejores prácticas de talento humano para direccionar estratégicamente las actividades de la organización de acuerdo a su misión y visión. Ante tal situación se formula la siguiente interrogante: ¿Cómo es la gestión del talento humano desde el enfoque estratégico en el sector minero de Venezuela, Colombia y Chile?

El sector minero, específicamente en las empresas Carbones del Guasare, Complejo Carbonífero de Cerrejón y Mina Invierno, actualmente se conciben como organizaciones privadas vinculadas a las acciones de la nación, con falencias en los enfoques estratégicos para gestionar con la gente, incidiendo en el direccionamiento estratégico que repercute en la gestión del talento humano del sector sujeto de estudio. Por tal motivo, en la investigación se analiza la gestión del talento humano desde el enfoque estratégico en el sector minero de Venezuela, Colombia y Chile. A tal efecto, la GTH desde el enfoque estratégico busca 
planificar, desarrollar, ejecutar, evaluar y controlar sistémicamente los procesos integrales de la organización, basada en la admisión, aplicación, compensación, desarrollo, retención y monitoreo de las personas, mediante iniciativas que aumenten tácticamente el rendimiento socio productivo de la empresa, alineados a sus principios corporativos. En este sentido, se hace necesario aplicar en las empresas del sector minero dicha variable de estudio, focalizando el rendimiento laboral que se puede desarrollar por el talento humano.

\section{OTROS ANTECEDENTES}

La GTH está orientada a las actividades operativas - administrativas, dotando a la empresa de colaboradores con competencias genéricas y técnicas transversales. Garantizar la ventaja derivada del factor humano se hace inimitable por la ambigüedad causal y complejidad social inherente a la integración de las mismas provocando en la competencia dificultades al replicar las transferencias de esas habilidades, conocimientos, aptitudes y actitudes que se desarrollan (Ramírez et al.,2018c; Safa et al., 2017). Las teorías actuales de la gestión humana han dejado de corresponder al dominio exclusivo de un área del conocimiento, ya sea la sociología o administración, se yuxtaponen en una mixtura de aproximaciones que desde diferentes ciencias y disciplinas se integran para producir un nuevo constructo teórico utilizado por la administración para abordar el sujeto de estudio: los actores de la organización.

Todo esto, da cuenta que la gestión humana debe posibilitar el establecimiento de la estrategia del negocio, creyendo en las personas como fuente de ventaja competitiva sostenible, cultura gerencial que comparta y defienda esta creencia, líderes de talento humano que entiendan las implicaciones del capital humano sobre las situaciones del negocio y puedan modificar el sistema de recursos humanos para solucionar esos problemas, concienciando que la función de la gestión humana actualmente es formar estrategas más que colaboradores pasivos (Caplan,2014).

Desde hace años el termino estrategia se ha empleado desde las operaciones militares hasta la actualidad, siendo el arte de proyectar y dirigir (Ramírez et al.,2018c; Caplan, 2014; Chiavenato, 2011; Münch, 2010; Chamberlain, 2010; Porter, 1980), definen criterios operacionales (Ansoff, 1991), en el área de la administración es el patrón o plan que incluye metas y políticas de la organización, coherentes a las acciones a cumplir (Gavino et al., 2012; Mintzberg et al., 1997). Su implicación en la gestión del talento humano, provee acciones encaminadas a consolidar un objetivo, garantizando recursos necesarios de la empresa para ejecutar lo cometido, involucrando al talento humano como socios estratégicos de la organización.

En estudios realizados de la gestión del talento humano por Cooke et al., (2014), Schiemann (2014) y Claussen et al., (2014), los resultados obedecen a la necesidad de adoptar un enfoque particularista versus a uno universalista, para conceptualizar y operacionalizar la gestión del talento en el contexto internacional. Al mismo tiempo para Caplan (2014) y Ramírez et al., (2018e), es importante crear un talento humano estratégico para la gestión incluyente y centrado en el desarrollo de talento, con implicaciones prácticas de la organización, proporcionando conocimiento para maximizar la efectividad del proceso empresarial.

En este sentido, los problemas y tópicos del quehacer de la gestión humana desde lo teórico-práctico, ha cambiado a lo largo del tiempo, centrándose en apoyar el desarrollo de las capacidades de los colaboradores, realizar una gestión por competencias y del conocimiento, dirigir de forma adecuada el talento humano, más que controlar, supervisar y simplemente administrar el personal con miras a obtener su rendimiento (Ahammad et al., 2012; Münch, 2010). Estas y otras tendencias, obedecen a procesos humanos integrados que visionen a la organización holísticamente, por lo que la gestión del talento humano posee un elemento estratégico en la empresa, trascendiendo en las actividades que realiza el colaborador, dando origen a procesos de cambio organizacional donde las personas son fundamentales.

La gestión del talento humano desde lo estratégico, es un campo de la teoría del management, forma parte del enfoque de la gerencia de recursos humanos, teóricamente se arraiga en el proceso histórico del desarrollo de la teoría de la organización, dentro de la perspectiva de la gestión de recursos humanos y capacidades (Escobedo y Andrade, 2017), orientada por su naturaleza y forma de dirigir estratégicamente el capital humano, mejorando el desempeño organizacional (Pantouvakis y Karakasnaki, 2017), expresado en términos de eficiencia, eficacia en el manejo de recursos, mejorando la productividad y rentabilidad de las empresas.

La globalización en las organizaciones donde predomina la competitividad, transferencia cultural, comercio internacional e inversión extranjera, determinan la consolidación de metas corporativas que impactan los logros propuestos por las empresas, favoreciendo la percepción oportuna de un mercado nuevo y especializarse en un segmento productivo que acompañados de calidad, mejoramiento tecnológico y eficacia de la producción, crean ventajas competitivas en los escenarios actuales, manteniendo así indicadores de altos desempeño que tributan al talento humano, el enfoque estratégico minimiza cualquier costo de error 
financiero/social que afecte la rentabilidad. Para Cuesta (2015), la gestión del talento humano desde el enfoque estratégico, está dada por las dimensiones estrategia organizacional, rasgos y tendencia actual, así como la necesidad del modelo funcional.

La estrategia organizacional es la creación, implementación y evaluación de las decisiones dentro de una organización, alcanzando objetivos a largo plazo, para ello deben socializarse las actividades, y que el personal participe en la creación de dichas estrategias (Fuentes y Cordón, 2016; Cuesta, 2015; Gupta y Shaw, 2014). Especifica la misión, visión y objetivos de la empresa, permiten el desarrollo de políticas y planes de acción relacionados a los proyectos y programas creados para lograrlos; asignando recursos para implementarlos (Ramírez y Ampudia, 2018a). Está relacionada con los estudios de la organización, se encarga de la dirección general empresarial, formulando estrategias que combinan: (a) análisis de la situación de la empresa, interna y externa, micro y macro ambiental, estudiando lo que sucede con sus competidores y cada parte interna de la empresa en sí; (b) fijación de objetivos, tanto a corto como a largo plazo, creando una visión y misión, para (c) desarrollar un plan estratégico.

Los rasgos y tendencia actual, facilitan las perspectivas teóricas de la gestión humana, como el centro de la organización, dentro de las cuales se encuentran las teorías actuales del diseño organizacional y psicología: organizaciones como sistema abierto y relaciones humanas. La relación entre el tamaño, diseño y cultura de la organización, con la concepción del adecuado manejo del ser humano en el espacio laboral (Ramírez et al., 2018c; Kim, et al., 2014; Meyers y Woerkom, 2014; León, 2011). Que la gente se identifique con las características, estilos emergentes y tendencias del talento humano, promovida en la organización (Cuesta, 2015). Hace posible que el personal adquiera nuevos aprendizajes, aptitudes, destrezas y formas de pensamiento que potencian su desarrollo en la empresa, propiciando un plan de carrera dentro de ella, formando el relevo generacional de los líderes.

Algunos rasgos y tendencias de gestión del talento humano que tanto en lo teórico como en la práctica cotidiana se consideran importantes, para el desarrollo de la gente son: el cambio organizacional, desarrollo humano, gestión del conocimiento - por competencias, tercerización (outsourcing), plan de carrera - sucesión, gerencia por procesos, cultura organizacional, marca personal y gestión internacional, alternativas novedosas que permiten a las organización enfrentarse al futuro, en la búsqueda de resultados cónsonos a la realidad y potencial competitivo (Ramírez et al.,2018c).

La necesidad del modelo funcional, establece normas en cada operación de la organización estableciendo comportamientos de los colaboradores ante el quehacer laboral. Expone el porqué de la existencia de la empresa, qué debe hacer y las metas específicas que la organización espera cumplir (Ramírez et al., 2018a 2018b; Bryant y Allen, 2013; Chiavenato, 2011; Wayne, 2010). Indica la ejecución de las funciones para la consolidación de objetivos, definición de oportunidades en función del comportamiento de los indicadores del sistema de gestión, dando respuesta a los planes de trabajo de la gestión estratégica del talento humano (Cuesta, 2015). Al mismo tiempo, operacionaliza las funciones, procesos, descubre necesidad de información, identifica oportunidades y establece una base para determinar el producto o servicio, atendiendo las condiciones de gasto.

Al abordar los referentes conceptuales de la gestión del talento humano desde lo estratégico, para quienes investigan permite hacer una triangulación de las dimensiones estrategia organizacional, rasgos y tendencia actual, y necesidad del modelo funcional, desarrollando en los colaboradores nuevos roles y retos en su actividad social, obedeciendo al soporte de la gestión humana para la sustentabilidad de la organización, propiciando gestión de conocimiento organizacional, garantizando un abordaje integral del humano en escenarios laborales, (ver tabla 1).

Tabla 1: Indicadores de la gestión del talento humano desde el enfoque estratégico

\begin{tabular}{|l|l|}
\hline Indicadores & Aspectos analizados \\
\hline $\begin{array}{l}\text { Estrategia } \\
\text { organizacional. }\end{array}$ & $\begin{array}{l}\text { Socialización de las actividades. } \\
\text { Ejecución de actividades. } \\
\text { Participación en la creación de las estrategias. }\end{array}$ \\
\hline $\begin{array}{l}\text { Rasgos y tendencia } \\
\text { actual. }\end{array}$ & $\begin{array}{l}\text { Identidad con las características promovidas para la gestión del talento humano. } \\
\text { Presencia de estilos emergente. } \\
\text { Implementación de tendencias actuales de talento humano. }\end{array}$ \\
\hline $\begin{array}{l}\text { Necesidad del } \\
\text { modelo funcional. }\end{array}$ & $\begin{array}{l}\text { Ejecución de las funciones para la consolidación de objetivos. } \\
\text { Definición de oportunidades en función del comportamiento de los indicadores del } \\
\text { sistema. } \\
\text { Respuesta del trabajo a la gestión estratégica del talento. }\end{array}$ \\
\hline
\end{tabular}




\section{METODOLOGÍA}

Enmarcado en el paradigma cuantitativo, de tipo descriptivo, diseño no experimental transeccional. Se utilizó la técnica de la encuesta, a través de un cuestionario estructurado por nueve (9) ítems, permitiendo estudiar la estrategia organizacional, rasgos y tendencia actual, y necesidad del modelo funcional, con escala ordinal de opciones de respuestas, (MDA): muy de acuerdo, (DA): de acuerdo, (I): indeciso, (ED): en desacuerdo, (MED): muy en desacuerdo, calificándose desde cinco (5) al uno (1) los enunciados positivos y del uno (1) al cinco (5) los enunciados negativos. De acuerdo al proceso de levantamiento de información en las unidades de análisis, después de aplicar el cuestionario sobre la población estudiada los datos fueron codificados y tabulados empleando el programa SPSS, versión 2.0, procesados e interpretados estadísticamente, utilizando la técnica de análisis descriptivo, de varianza y regresión lineal, con la correspondiente discusión teórica de los resultados, permitiendo llegar a las conclusiones.

Los datos por esta vía recolectados, previo al análisis exploratorio de los mismos, (Curtosis, asimetría, prueba de Kolmogorov-Smirnof y Caja y Bigotes) y demostrado el cumplimiento de condicionantes, estableció el estatus y preponderancia de las dimensiones estudiadas aplicando el análisis de la varianza (ANOVA) con la respectiva prueba de medias (Tukey), para el análisis de los resultados se empleó un baremo de categorización (ver tabla 2). La validez del contenido del instrumento, se sometió a revisión de diez (10) expertos en metodología y gerencia del talento humano. Se realizó una prueba piloto en un número de sujetos similares a la población, una vez recopilada la información se aplicó la fórmula de confiabilidad Alfa de Cronbach, dando como resultado un $0,91 \mathrm{r}$, indicando que el instrumento es altamente confiable.

Tabla 2: Baremo de categorización de la media aritmética estableciendo el comportamiento de la variable gestión estratégica de talento humano

\begin{tabular}{|l|l|}
\hline Rango de puntaje & Categorización \\
\hline $1-1,79$ & Pésimo. \\
\hline $1,80-2,59$ & Moderadamente pésimo. \\
\hline $2,6-3,39$ & Moderado. \\
\hline $3,4-4,19$ & Moderadamente óptimo. \\
\hline $4,2-5$ & Óptimo. \\
\hline
\end{tabular}

Los criterios de selección de las unidades de análisis: Carbones del Guasare, S.A de Venezuela; el Complejo Carbonífero de Cerrejón en Colombia y Mina Invierno de Santiago de Chile, obedece por su similar: (a) naturaleza y razón social al pertenecer al sector minas, (b) capital suscrito, (c) total de activos y pasivos, (d) cantidad de colaboradores vinculados fijos, (e) tamaño de las empresas y (f) modelo y estructura de gerencias adscritas a la vice presidencia. La población de estudio, compuesta por cincuenta y cinco (55) gerentes, todos mayores de edad, personal fijo, cargo gerencial, indiferentemente de su estado civil, de cada vice presidencia: financiera, sostenibilidad y asuntos públicos, operaciones, financiera y comercial.

La metodología se centró mediante: (a) la selección del tema, revisión bibliográfica, a fin de estructurar planteamiento del problema, referentes teóricos, justificación y objetivo; (b) elaboración de la metodología; (c) construcción del instrumento, sometido a prueba de expertos para precisar la validez del mismo y elaborar la versión final para la posterior aplicación a la población de estudio; (d) tratamiento estadístico de análisis y discusión de los resultados; (e) y obtención de conclusiones y recomendaciones. Se incluyen actividades sugeridas de acuerdo al tipo, nivel y diseño de la investigación, propuestas por Bernal (2016), Bryman (2015), Hernández, Fernández y Baptista (2014), Hernández (2010), Lerma (2009) y Hurtado (2008).

\section{RESULTADOS Y DISCUSIÓN}

Al analizar la gestión del talento humano desde el enfoque estratégico (ver tabla 3), se desagregan los elementos definitorios de los indicadores desde la teoría empleada por Cuesta (2015). En la Tabla 3, (ns) =no significativa, $\left({ }^{*}\right)=$ significativa, $\left({ }^{* *}\right)=$ Altamente significativa. Además, letras iguales entre las medias de un aspecto es equivalente a (ns). Respecto a la estrategia organizacional: socialización de las actividades, ejecución de actividades y participación en la creación de las estrategias, no existen diferencias estadísticamente significativas $(F=2,2 p>0.12)$, en consecuencias todas están en un mismo nivel de ejecución, pudiéndose considerar éste como moderadamente óptimo en razón que las medias están entre 3,4-4,19.

Estos resultados se asemejan a la investigación efectuada por Gupta y Shaw (2014) y Ambrosius (2016), obedeciendo al empleo incipiente de las estrategias, sin embargo, por su importancia deben diseñarse procedimientos gerenciales para que su nivel sea óptimo. Un aspecto positivo a destacar que a pesar de no 
estar en un nivel óptimo las mismas se encuentran parejas, dándoles el mismo nivel de importancia. La estrategia organizacional se caracteriza por una visión del futuro, activa y anticipadamente, aporta un marco a la dirección unificada de la empresa en función de sus metas (Ramírez y Ampudia, 2018a; Fuentes y Cordón, 2016; Cuesta, 2015). Orientando los recursos, para el desarrollo de ventajas competitivas, indica la necesidad de adaptación sistemática del entorno, enfatiza encontrar posiciones favorables, considerando acciones futuras en los escenarios, con un impacto final importante.

Tabla 3: Análisis de la varianza para las medias de los puntajes de aspectos constitutivos de los indicadores del enfoque estratégico y prueba de medias

\begin{tabular}{|c|c|c|c|c|}
\hline Indicadores & Aspectos analizados & Media & $F$ & $p$ \\
\hline \multirow{3}{*}{$\begin{array}{l}\text { Estrategia } \\
\text { organizacional. }\end{array}$} & Socialización de las actividades. & 3.94 (a) & \multirow{3}{*}{2,2} & \multirow{3}{*}{0,12 (ns) } \\
\hline & Ejecución de actividades. & $3.58(\mathrm{a})$ & & \\
\hline & Participación en la creación de las estrategias. & $3.54(\mathrm{a})$ & & \\
\hline \multirow[t]{3}{*}{$\begin{array}{l}\text { Rasgos y tendencia } \\
\text { actual. }\end{array}$} & $\begin{array}{l}\text { Identidad con las características promovidas para la } \\
\text { gestión del talento humano. }\end{array}$ & $3(a)$ & \multirow{3}{*}{7,15} & \multirow{3}{*}{$0.001\left({ }^{* *}\right)$} \\
\hline & Presencia de estilos emergente. & $2.4(\mathrm{~b})$ & & \\
\hline & $\begin{array}{l}\text { Implementación de tendencias actuales de talento } \\
\text { humano. }\end{array}$ & $2.14(b)$ & & \\
\hline \multirow[t]{3}{*}{$\begin{array}{l}\text { Necesidad del } \\
\text { modelo funcional. }\end{array}$} & $\begin{array}{l}\text { Ejecución de las funciones para la consolidación de } \\
\text { objetivos. }\end{array}$ & $3.05(a)$ & \multirow{3}{*}{0.662} & \multirow{3}{*}{$0.5(\mathrm{~ns})$} \\
\hline & $\begin{array}{l}\text { Definición de oportunidades en función del } \\
\text { comportamiento de los indicadores del sistema. }\end{array}$ & $3(a)$ & & \\
\hline & $\begin{array}{l}\text { Respuesta del trabajo a la gestión estratégica del } \\
\text { talento. }\end{array}$ & $2.78(\mathrm{a})$ & & \\
\hline
\end{tabular}

En cuanto a los rasgos y tendencia actual, fueron analizados en función a la identidad con las características promovidas para la gestión del talento humano, presencia de estilos emergentes e implementación de tendencias actuales de talento humano, encontrándose que existen diferencias altamente significativas en su implementación que el valor de $\mathrm{F}=7.15$ es altamente significativo a $\mathrm{p} \leq 0.001$. La prueba de múltiples rangos de Tukey permitió detectar la diferencia, la identidad con las características promovidas para la gestión del talento humano con una media de 3 siendo moderada, difiere estadísticamente de la valoración hecha a la presencia de estilos emergente e implementación de tendencias actuales de talento humano que no difieren entre si y al tener medias entre 2.4 y 2.14 respectivamente pueden ser categorizadas como moderadamente pésima, de acuerdo al rango de puntaje 1,80-2,59.

En este sentido se hace necesario valorar la identidad, y considerar los estilos emergentes para la implementación de tendencias actuales de talento humano, estos hallazgos son afines a los resultados de las investigaciones realizadas por Ramírez et al., (2018c), Pantouvakis y Karakasnaki (2017), Meyers y Woerkom (2014) y León (2011), donde estos aspectos son necesarios para promover en las organizaciones la gestión estratégica del talento humano, favoreciendo la visión estratégica de la empresa, propiciando organizaciones felices, con igualdad de género, tendencias verdes, economía naranja, a lo natural, emergente, original, técnico profesional y sistémico. Los rasgos y tendencia actual, en la gestión del talento humano, especifican las características de la organización, afectadas por nuevas formas de acompañar a la gente. De acuerdo a Ramírez et al., (2018d), Cuesta (2015), Kim et al., (2014), muestra el cambio profundo que enfrentan los líderes empresariales a nivel mundial, provocando el surgimiento de la empresa social o social enterprise.

De acuerdo a la necesidad del modelo funcional, se evidencia que en la ejecución de las funciones para la consolidación de objetivos, definición de oportunidades en función del comportamiento de los indicadores del sistema y respuesta del trabajo a la gestión estratégica del talento, no existen diferencias significativas entre sus promedios de ponderación $(F=0,66 p>0.5)$ por tanto su status en las organizaciones están en un mismo nivel, en razón a la ubicación de las medias en el rango comprendido entre 2,6-3.39 es moderado. Estos resultados, son similares a los expuestos por la investigación de Schiemann, (2014), por tal motivo como estrategia organizacional se debe tomar en cuenta este indicador a fin de ser óptimo, utilizándose posteriormente como un elemento competitivo en el sector real.

La necesidad del modelo funcional, son las actividades, procesos y operaciones en el sistema o modelo del área laboral, donde se podrían identificar posibles oportunidades de mejoras (Ramírez et al., 2018b; Safa et al., 2017; Cuesta, 2015; Bryant y Allen, 2013; Chiavenato, 2011; Wayne, 2010). Al observar los resultados, el alcance del indicador no es óptimo, por tal motivo se hace necesario reducir oportunamente las brechas de conocimiento originadas por este en las áreas funcionales. Para el análisis de la gestión del talento humano desde el enfoque estratégico, se hace necesario analizar lo que ocurre integralmente con sus indicadores: 
estrategia organizacional, rasgos y tendencia actual, y la necesidad del modelo funcional. Se evidencia diferencias altamente significativas $(F=3,26 ; p=0.0001)$ considerándose como moderadamente óptimo, predominando la estrategia organizacional con una media 3.7, siendo moderadamente óptimo, seguido de los rasgos y tendencia actual con la media de 2.95, moderada, y el indicador más deprimido, la necesidad del modelo funcional que al tener una media de 2.52 es moderadamente pésimo (ver tabla 4). En la Tabla 4, (ns) $=$ no significativa, $\left({ }^{*}\right)=$ significativa, $\left({ }^{* *}\right)=$ Altamente significativa. Las letras iguales entre las medias de un aspecto son equivalentes a (ns). Estos resultados evidencian la desactualización de las dinámicas actuales de la gerencia estratégica en términos de GTH, adaptada a la necesidad del modelo funcional organizacional.

Tabla 4: ANOVA para las medias de los indicadores de la GTH desde el enfoque estratégico y prueba de medias

\begin{tabular}{|l|l|l|l|l|}
\hline Variable & Indicadores & Media & $F$ & $p$ \\
\hline \multirow{4}{*}{ GTH desde el enfoque estratégico } & Estrategia organizacional. & $3.7(\mathrm{a})$ & & \\
\cline { 2 - 3 } & Rasgos y tendencia actual. & $2.95(\mathrm{~b})$ & \multirow{3}{*}{3.26} & \multirow{3}{*}{$0,0001\left(^{* *}\right)$} \\
\cline { 2 - 3 } & Necesidad del modelo funcional. & $2.52(\mathrm{c})$ & & \\
\hline
\end{tabular}

Ante estos resultados, investigaciones de Ramírez et al., (2018c), Safa et al., (2017), Cuesta (2015), Ambrosius (2016), Caplan (2014), Kim et al., (2014), muestran su similitud, que a pesar que los hallazgos son moderadamente óptimos las diferencias son altamente significativas, descuidando indicadores que podrían desfavorecer la GTH. La gestión del talento humano desde el enfoque estratégico, sienta las bases para la ventaja competitivas de la organización, mediante la creación de una estructura eficaz, talento calificado, diseño, proposición, sistemas de pensamiento, cultura y valor de mano de obra (Bryant y Allen, 2013; Cuesta 2015; Ambrosius, 2016; Ramírez et al., 2018c; Safa et al., 2018). Ante tales aportes, al considerarse como moderadamente óptimo la gestión del talento humano desde el enfoque estratégico en el sector minero de Venezuela, Colombia y Chile, posibilita en éstas el desarrollo de habilidad, pertinencia del talento en el área requerida y productividad a la organización.

Estos resultados evidencian, los retos de las organizaciones para garantizar en la gestión del talento humano la estrategia organizacional, adaptarse a los rasgos y tendencia actual, de acuerdo a la necesidad del modelo funcional, mediado por los principios y objetivos corporativos, adecuado al contexto global, afectado por factores políticos, sociales, económicos y hasta bélicos, convirtiéndose en una guía para alinear los procesos empresariales, garantizado el adecuado uso de los recursos, con miras a lo sustentable y sostenible.

\section{DISCUSIÓN FINAL}

Se concluye que la gestión del talento humano desde el enfoque estratégico es moderadamente óptimo, adaptándose a las nuevas necesidades del entorno con miras a lo conceptual y creativo, donde la estrategia responde a la demanda del mercado, creando un valor en la gente como factor diferenciador para competir en la nueva economía de la oferta y demanda, adoptando prácticas de gestión sustentable que garantice la rentabilidad y permanencia de la empresa en el mundo, adecuada a las dinámicas de la naturaleza del sector.

La estrategia organizacional está en un nivel moderadamente óptimo, sin predominio de ningunos de sus indicadores por tanto se requiere de un esfuerzo sistémico de gestión en las organizaciones para aplicar equitativa e igualmente esta dimensión, permitiendo un equilibrio cónsono a la realidad de cada empresa, armonizando cada subsistema de talento humano, propiciando rentabilidad y permanencia en el mercado. De acuerdo a los rasgos y tendencias actual, es considerado como moderado, apuntan al favorecimiento de la identidad con las características promovidas para la gestión de gente, por encima de la presencia de estilos emergente e implementación de tendencias actuales de talento humano, impactando socialmente en el comportamiento de los indicadores del sistema, los cuales se encuentran deprimidos en las empresas estudiadas ya que su nivel considerado es moderadamente pésimo, en consecuencia, disminuye la posibilidad de adaptarse a nuevas tendencias emergentes para la GTH.

La necesidad de un modelo funcional es moderadamente pésima, requiriendo la pronta intervención del mismo en procura de ejecutar adecuadamente las actividades, definir oportunidades para convertirlas en fortalezas, dando respuestas a la gestión estratégica del talento humano. En este sentido, existe un predominio de la ejecución de la estrategia organizacional sobre la atención a la necesidad de aspectos vinculados a los rasgos y tendencias actuales de la GTH y necesidad de establecer un modelo funcional siendo perentorio. El adoptar la gestión del talento humano desde el enfoque estratégico, responde al cómo se logrará lo planeado con prácticas innovadoras, para ello, se alinean los propósitos tanto del colaborador como el de la organización, consolidando integralmente las metas empresariales de intereses en común concertados. Ubicando estratégicamente al talento humano en puesto medulares de acuerdo a sus competencias, involucrando activamente a su personal en la 
cadena socio productiva, generando valor agregado, garantizando la optimización sistémica de los recursos: humanos, físicos y económicos. Todo esto impacta la fidelización de sus clientes, capacidad relacional con sus redes corporativas, stakeholder, clúster, y atracción de nuevos talentos que buscan la gestión de conocimiento.

\section{CONCLUSIONES}

De acuerdo a los resultados obtenidos, se concluye que: (1) en el sector minero de Venezuela, Colombia y Chile se le da prioridad a la estrategia organizacional, fortalecido por el indicador socialización de las actividades laborales, permitiendo articular sistémicamente las acciones mediadas por sus objetivos para consolidarlas; (2) además se encontró que entre los aspectos analizados, son determinantes para la gestión del talento humano desde el enfoque estratégico principalmente: la identidad de la gente en correspondencia a la cultura organizacional y su ejecución de las funciones para el cumplimiento de metas; y (3) el impacto de la prueba de medias analizados se ve influenciada por las teorías, escuelas, tendencias administrativas, modelos del comportamiento organizacional y época, de acuerdo a los diversos enfoques que asume el talento humano.

\section{REFERENCIAS}

Ahammad, M. F., K. W. Glaister., Y. Weber., y S.Y. Tarba, Top management retention in cross-border acquisitions: The roles of financial incentives, acquirer's commitment and autonomy, doi: 10.1504/EJIM.2012.048158, European Journal of International Management, 6(4), 458 - 480, (2012)

Ambrosius, J., Strategic talent management in emerging markets and its impact on employee retention: Evidence from Brazilian MNCs, doi: 10.1002/tie.21799, Thunderbird International Business Review, 60(1), 53-68, (2016)

Anaya, T. J., Organización de la producción industrial. Un enfoque de gestión operativa en la fábrica, $1^{\text {ra }}$ ed., 001-208, Esic Editorial, Madrid, España, (2016)

Ansoff, H.I., Critique of Henry Mintzberg's The design school: Reconsidering the basic premises of strategic management, doi: 10.1002/smj.4250120605, Strategic Management Journal, 12(6), 449-461, (1991)

Bernal, T.C., Metodología de la investigación, $4^{\text {ta }}$ ed., 001-384, Editorial Pearson, Bogotá, Colombia, (2016)

Bryant, P. C., y D. G. Allen, Compensation, benefits and employee turnover: HR strategies for retaining top talent, doi: 10.1177/0886368713494342, Compensation \& Benefits Review, 45(3), 171-175, (2013)

Bryman, A., y E. Bell, Business research methods, $4^{\text {th }}$ ed., 001-778, Oxford University Press, Oxford, England, (2015)

Caplan, J., Thinking differently about talent development: An interview with Janice Caplan, author of Strategic Talent Development, doi: 10.1108/HRMID-03-2014-0034, Human Resource Management International Digest, 22(2), 42-45, (2014)

Claussen, J., T. Grohsjean., J. Luger., y G, Probst, Talent management and career development: What it takes to get promoted, doi: 10.1016/j.jwb.2013.11.007, Journal of World Business, 49(1), 236-244, (2014)

Chamberlain, G.P., La Comprensión De La Estrategia, 2da ed., 001-218, CreateSpace, Charleston, (2010)

Chiavenato, I. Administración de recursos humanos El capital humano de las organizaciones, 9na ed.,001-736, Editorial McGraw - Hill, México, (2011)

Coff, R., y D. Kryscynski, Drilling for micro-foundations of human capital based competitive advantages, doi: 10.1177/0149206310397772, Journal of Management, 37(5), 1429-1433, (2011)

Cooke, F; D. Saini., y J. Wang, Talent management in China and India: A comparison of management perceptions and human resource practices, doi: 10.1016/j.jwb.2013.11.006, J. of world business, 49 (2), 225-235, (2014)

Cuesta, S.A., Gestión del talento humano y del conocimiento, 5ta ed., 003-437, Ediciones ECOE, Bogotá, Colombia, (2015)

Deloitte., Tendencias Globales del Capital Humano, (2018)

Escobedo, G.G. y V.M. Andrade, Desarrollo sustentable. Estrategia en las empresas para un futuro mejor, $1^{\text {ra }}$ ed., 001 381, Alfaomega grupo editor, México, (2017)

Fuentes, F.M. y P.E. Cordón, Fundamentos de Dirección y Administración de Empresas, $3^{\text {ra }}$ ed.,001-272, Ediciones Pirámide (Grupo Anaya, S.A), España, (2016)

Gavino, M. C., S. J. Wayne., y B. Erdogan, Discretionary and transactional human resource practices and employee outcomes: The role of perceived organizational support, doi: 10.1002/hrm.21493, Human Resource Management, 51(5), $665-686,(2012)$

Gupta, N., y J. D. Shaw, Employee compensation: The neglected area of HRM research, doi: 10.1016/j.hrmr.2013.08.007, Human Resource Management Review, 24(1), 1-4, (2014)

Hernández, S.R., Metodología de la investigación, $5^{\text {ta }}$ ed., 89-101, Editorial McGraw - Hill, México, (2010) 
Hernández, R.F., C. Fernández., y M. Baptista, Metodología de la investigación, 6 ${ }^{\text {ta }}$ ed.,001-607, Editorial McGraw - Hill, México, (2014)

Hurtado de B.J., Cómo formular objetivos de investigación: un acercamiento desde la investigación holística, $2^{\text {da }}$ ed., 01 75, Ediciones Gavilán C.A, Caracas, Venezuela, (2008)

Kim, Y; R. Williams., W. Rothwell., y P. Penaloza, A strategic model for technical talent management: a model based on a qualitative case study, doi: 10.1002/piq.21159, Performance improvement quarterly, 26(4), 93-121, (2014)

León, S.G., Nuevos enfoques para la gestión estratégica de la I+D e innovación en las universidades, ISSN: 0034-592X, Revista de Educación,382(355), 83-108, (2011)

Lerma G.H., Metodología de la investigación: propuesta, anteproyecto y proyecto, $5^{\text {ta }}$ ed., 01-190, Ecoe Ediciones, Bogotá, Colombia, (2009)

ManpowerGroup. Estudio sobre escasez de talento, (2018)

Maxwell, C. J. Desarrolle el líder que está en usted, $7^{\mathrm{ma}}$ ed., 001-200, San Pablo, Bogotá - Colombia, (2016)

Meyers, M. C. y M. Woerkom, The influence of underlying philosophies on talent management: Theory, implications for practice, and research agenda, doi: 10.1016/j.jwb.2013.11.003, J. of World Business, 49(1), 192-203, (2014)

Mintzberg, H., J.B. Quinn., y J. Voyer, El proceso estratégico: conceptos, contextos y casos, 1ra ed., 001-641, Pearson Educación, Naucalpan de Juárez, Estado de México, (1997)

Münch L., Administración, gestión organizacional enfoques y procesos administrativos, $3^{\text {ra }}$ ed., 01-320, Pearson Educación, Naucalpan de Juárez, Estado de México, (2010)

Pantouvakis, A y M. Karakasnaki, Role of the human talent in total quality management-performance relationship: an investigation in the transport sector, doi: 10.1080/14783363.2017.1303873, Total Quality Management \& Business Excellence, 28(1), 959-973, (2017)

Porter, M.E., Competitive strategy: Techniques for analyzing industries and competitors, $1^{\text {ra }}$ ed., 001-396, Free Press, New York. (1980)

Ramírez, R.I., y A. Hugueth, Modelo de comunicación productiva para las organizaciones de salud pública en Venezuela, ISSN: 1012-1587, Revista Opción, 33(83), 305-335, (2017)

Ramírez, R. y D, Ampudia, Factores de competitividad empresarial en el sector comercial, ISSN: 2443-4426, Revista Electrónica de Ciencia y Tecnología del Instituto Universitario de Tecnología de Maracaibo (RECITIUTM), 4(1), 16-32, (2018a)

Ramírez, R.I, I. Avendaño, L. Alemán, C. Lizarazo, R. Ramírez., y Y. Cardona, Principles of social responsibility for the strategic management of the talent human public health organizations, ISSN: 0798-1015, Revista Espacios, 39(37), 2227, (2018b)

Ramírez, R. I., H.C. Chacón, y O. N. El Kadi, Gestión estratégica del talento humano en las PYMES, 1ra ed., 001-120, Editorial Corporación CIMTED Medellín, Colombia, (2018c)

Ramírez R.I., N.D. Lay Raby., I. Avendaño., y B.A. Herrera, Liderazgo resiliente como competencia potenciadora del talento humano, ISSN: 1012-1587, Revista Opción, 34(86), 826-854, (2018d)

Ramírez, R.I., J.V. Villalobos y B.A. Herrera, Proceso de talento humano en la gestión estratégica, ISSN: 1012-1587, Revista Opción,34(18), 2076-2101, (2018e)

Safa, M; Weeks, K., R. Stromberg., y A.A. Azam., Strategic Port Human Resource Talent Acquisition and Training: Challenges and Opportunities., Strategic port human resource talent acquisition and training: challenges and opportunities, Springer Nature Switzerland AG, Volume 594, pp 205-215, California, USA (2017)

Schuler, R., y I. Tarique, Global talent management: Theoretical perspectives, systems, and challenges., Handbook of research in international human resource management, pp 205-219, Cheltenham, England: Edward Elgar, (2012)

Schiemann, W. A., From talent management to talent optimization. Journal of World Business, doi: 10.1016/j.jwb.2013.11.012, 49(2), 281 - 288, (2014)

Villalobos, J. V., y R.I. Ramírez, El derecho a la autobiografía: dimensión ius-filosófica desde la perspectiva de $H$. Arendt y P. Ricoeur, ISSN: 1012-1587, Revista Opción,34(18), 1012-1587, (2018)

Wayne, M.R. Administración de recursos humanos, 11 va ed., 001-512, Pearson Educación de México, S.A, México, (2010) 
